University of Nebraska - Lincoln

DigitalCommons@University of Nebraska - Lincoln

John R. Hardy Papers

Research Papers in Physics and Astronomy

2003

\title{
Spectral Functions of the Falicov-Kimball Model with Electronic Ferroelectricity
}

Wei-Guo Yin

University of Nebraska, Omaha, Nebraska

Wai-Ning Mei

University of Nebraska at Omaha, physmei@unomaha.edu

Chun-Gang Duan

University of Nebraska at Omaha, cgduan@clpm.ecnu.edu.cn

Hai-Qing Lin

The Chinese University of Hong Kong, Hong Kong, People's Republic of China

John R. Hardy

University of Nebraska - Lincoln

Follow this and additional works at: https://digitalcommons.unl.edu/physicshardy

Part of the Physics Commons

Yin, Wei-Guo; Mei, Wai-Ning; Duan, Chun-Gang; Lin, Hai-Qing; and Hardy, John R., "Spectral Functions of the Falicov-Kimball Model with Electronic Ferroelectricity" (2003). John R. Hardy Papers. 66.

https://digitalcommons.unl.edu/physicshardy/66

This Article is brought to you for free and open access by the Research Papers in Physics and Astronomy at DigitalCommons@University of Nebraska - Lincoln. It has been accepted for inclusion in John R. Hardy Papers by an authorized administrator of DigitalCommons@University of Nebraska - Lincoln. 


\title{
Spectral functions of the Falicov-Kimball model with electronic ferroelectricity
}

\author{
Wei-Guo Yin, ${ }^{1} *$ W. N. Mei, ${ }^{1}$ Chun-Gang Duan, ${ }^{1}$ Hai-Qing Lin, ${ }^{2}$ and J. R. Hardy ${ }^{3}$ \\ ${ }^{1}$ Department of Physics, University of Nebraska, Omaha, Nebraska 68182, USA \\ ${ }^{2}$ Department of Physics, The Chinese University of Hong Kong, Hong Kong, People's Republic of China \\ ${ }^{3}$ Department of Physics and Center for Electro-Optics, University of Nebraska, Lincoln, Nebraska 68588, USA
}

(Received 26 February 2003; published 19 August 2003)

\begin{abstract}
We calculate the angular resolved photoemission spectrum of the Falicov-Kimball model with electronic ferroelectricity where $d$ - and $f$-electrons have different hoppings. In mix-valence regimes, the presence of strong scattering processes between $d-f$ excitons and a hole, created by emission of an electron, leads to the formation of pseudospin polarons and novel electronic structures with bandwidth scaling with that of $d-f$ excitons. Especially, in the two-dimensional case, we find that flat regions exist near the bottom of the quasiparticle band in a wide range of the $d$ - and $f$-level energy difference.
\end{abstract}

DOI: 10.1103/PhysRevB.68.075111

PACS number(s): 71.28. $+\mathrm{d}, 71.27 .+\mathrm{a}, 77.80 .-\mathrm{e}$

Ferroelectric materials have long been important to physical research and technological application. Besides commonly known displacive and order-disorder mechanisms, ${ }^{1}$ recently the idea of electronic ferroelectricity (EFE) has been attracting considerable attention. EFE was introduced by Portengen et al. ${ }^{2,3}$ in their mean field theory of an extended Falicov-Kimball model (FKM). ${ }^{4}$ The FKM, having a long successful history in dealing with correlated electron systems, ${ }^{5}$ was then used to model a system that consists of two bands of different parity, say itinerant $d$ electrons and localized $f$ orbitals, in which mix-valence states may occur depending on the $d-f$ coupling strength. Portengen et al. pointed out that inclusion of a $d-f$ hybridization in the FKM could give rise to a spontaneous electric polarization due to a Bose-Einstein condensation (BEC) of $d-f$ excitons when the excitation energy goes to zero at the critical value of the $f$-level energy. ${ }^{2,3}$ It is expected that such a purely electronic mechanism would provide fascinating physical features which are desirable for many applications; for instance, the static dielectric constant in an electronic ferroelectric could exceed $10^{4}{ }^{3}$

However, EFE in the FKM is still controversial after tested by different theoretical treatments of the underlying strongly correlated electron system. ${ }^{6-10}$ Most recently, Batista proposed a new extension of the FKM in which an $f-f$ hopping was included. ${ }^{11}$ Mapping the strong coupling limit of this model into an $x x z$ pseudospin 1/2 model with a magnetic field along the $z$-axis and supported by quantum Monte Carlo calculations performed earlier by Schmid et al. ${ }^{12}$ he showed that a BEC of $d-f$ excitons does exist in the phase diagram. ${ }^{11}$

The purpose of this paper is to present a theoretical study of the angular resolved photoemission spectrum of this extended FKM. In the pseudospin picture, ${ }^{11}$ we study the spectral functions of one hole, created by emission of one electron, in an extended $t-J$ model with different hoppings for each pseudospin flavor. The problem of single hole motion in a local (pseudo)spin background has become an essential issue in understanding anomalous physical properties of high temperature superconductors ${ }^{13-15}$ and of colossal magnetoresistance manganites. ${ }^{16,17}$ It has been demonstrated that quantum antiferromagnetic (pseudo)spin fluctuations have strong impact on the low-energy scale physics of these materials. In this paper, we show that strong scattering processes between the hole and the $d-f$ excitons take place even in the ferroelectric regime that has a ferromagnetic pseudospin configuration, thus give rise to the formation of pseudospin polarons and novel electronic structures.

The extended Falicov-Kimball model for spinless fermions on a hypercubic lattice is ${ }^{11}$

$$
\begin{aligned}
H= & \epsilon_{d} \sum_{\mathbf{i}} n_{\mathbf{i}}^{d}+\epsilon_{f} \sum_{\mathbf{i}} n_{\mathbf{i}}^{f}+t_{d} \sum_{\langle\mathbf{i}, \mathbf{j}\rangle} d_{\mathbf{i}}^{\dagger} d_{\mathbf{j}}+t_{f} \sum_{\langle\mathbf{i}, \mathbf{j}\rangle} f_{\mathbf{i}}^{\dagger} f_{\mathbf{j}} \\
& +U^{f d} \sum_{\mathbf{i}} n_{\mathbf{i}}^{d} n_{\mathbf{i}}^{f},
\end{aligned}
$$

where $n_{\mathbf{i}}^{d}=d_{\mathbf{i}}^{\dagger} d_{\mathbf{i}}$ and $n_{\mathbf{i}}^{f}=f_{\mathbf{i}}^{\dagger} f_{\mathbf{i}}$ are the occupation numbers of $d$ - and $f$-orbitals, respectively. Representing the two orbital flavors by a spin-1/2 variable, $c_{\mathbf{i} \uparrow}=d_{\mathbf{i}}$ and $c_{\mathbf{i} \downarrow}=f_{\mathbf{i}}$, and using the notion of the pseudospin operator $\boldsymbol{\tau}_{\mathbf{i}}=\Sigma_{\mu \nu} c_{\mathbf{i} \mu}^{\dagger} \boldsymbol{\sigma}_{\mu \nu} c_{\mathbf{i} \nu}$ with $\left\{\boldsymbol{\sigma}_{\mu \nu}\right\}$ being the Pauli matrices, one can derive an effective $t-J$ model, $H_{\mathrm{eff}}=H_{t}+H_{J}$, in the strong coupling limit of (1) near half-filling, ${ }^{11}$

$$
\begin{gathered}
H_{J}=\sum_{\langle\mathbf{i}, \mathbf{j}\rangle}\left[J_{z} \tau_{\mathbf{i}}^{z} \tau_{\mathbf{j}}^{z}+J_{\perp}\left(\tau_{\mathbf{i}}^{x} \tau_{\mathbf{j}}^{x}+\tau_{\mathbf{i}}^{y} \tau_{\mathbf{j}}^{y}\right)\right]+B_{z} \sum_{\mathbf{i}} \tau_{\mathbf{i}}^{z}, \\
H_{t}=\sum_{\langle\mathbf{i}, \mathbf{j}\rangle, \sigma} t_{\sigma}\left(\widetilde{c}_{\mathbf{i} \sigma}^{\dagger} \widetilde{c}_{\mathbf{j} \sigma}+\widetilde{c}_{\mathbf{j} \sigma}^{\dagger} \widetilde{c}_{\mathbf{i} \sigma}\right),
\end{gathered}
$$

where $\widetilde{c}_{\mathbf{i} \sigma}=c_{\mathbf{i} \sigma}\left(1-n_{\mathbf{i} \sigma}\right)$ is the constrained fermion operator, $t_{\uparrow}=t_{d}, t_{\downarrow}=t_{f}, J_{z}=2\left(t_{\uparrow}^{2}+t_{\downarrow}^{2}\right) / U^{f d}, J_{\perp}=4 t_{\uparrow} t_{\downarrow} / U^{f d}$, and $B_{z}$ $=\epsilon_{d}-\epsilon_{f}$ is the $d$ - and $f$-level energy difference acting as a magnetic field along the $z$ direction.

To calculate the spectral functions of one hole in the FKM, we need to diagonalize half-filled $H_{\text {eff }}$ first, to which only $H_{J}$ contributes. We employ linear spin wave theory to diagonalize $H_{J}$. To examine the reliability of linear spin wave theory, we compare our phase diagram at half-filling with that obtained by others ${ }^{11,12,18}$ in the following. We find that the ground state of $H_{J}$ is one of the following phases: full $d$-band, full $f$-band, staggered orbital ordering, and the BEC of $d-f$ excitons that is ferroelectric for $J_{\perp}<0$ or anti- 


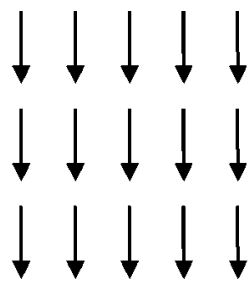

(a)

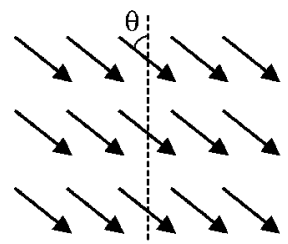

(c)

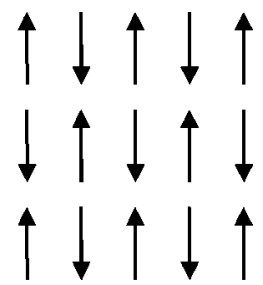

(b)

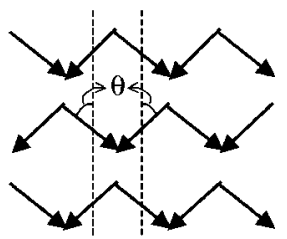

(d)
FIG. 1. Schematic pictures of the pseudospin configuration in (a) full $f$-band, (b) staggered orbital ordering, (c) ferroelectric canted state, and (d) antiferroelectric canted state.

ferroelectric for $J_{\perp}>0$. The BEC of $d-f$ excitons has partial magnetization, whose pseudospin configuration is assumed to be a canted state with respect to the $z$ axis [Fig. 1(c) or $1(\mathrm{~d})$ ], where the canting angle

$$
\cos \theta=\frac{\left|B_{z}\right|}{z S\left(J_{z}+\left|J_{\perp}\right|\right)},
$$

where $S=1 / 2$ is the value of the pseudospins and $z$ is the coordination number. Indeed, the spin excitation (i.e., $d-f$ exciton) in the canted state is a gapless Goldstone mode which corresponds to a uniform precession of the pseudospins around the $z$ axis. We obtain the following $J_{z^{-}}\left|B_{z}\right|$ phase diagram: a critical line of a second-order transition between the BEC of $d-f$ excitons and full $d$ - or $f$-band is $\left|B_{z}\right|=z S\left(J_{z}+\left|J_{\perp}\right|\right)$, while for $J_{z}>\left|J_{\perp}\right|$ there is another critical line of a first-order transition between staggered orbital ordering and the BEC of $d-f$ excitons, $\left|B_{z}\right|=z S \sqrt{J_{z}^{2}-J_{\perp}^{2}}$. We present the two-dimensional (2D) phase diagram in Fig. 2 , compared with results from quantum Monte Carlo studies on finite size systems up to $96 \times 96 .^{12}$ Our results agree well with the quantum Monte Carlo studies. Notice that in Fig. 2 the borderline between staggered orbital ordering and the BEC of $d-f$ excitons obtained from linear spin wave theory (solid line, $\left|B_{z}\right|=z S \sqrt{J_{z}^{2}-J_{\perp}^{2}}$ ) does not perfectly coincide with that from the Monte Carlo calculations (dashed line, $\left|B_{z}\right| \simeq 0.88 z S \sqrt{J_{z}^{2}-J_{\perp}^{2}}$ for $\left.1 \leqslant J_{z} /\left|J_{\perp}\right| \leqslant 3.5\right)$. It appears that linear spin wave theory overestimates the stability of the staggered orbital ordering, and this might be remedied by higher order spin wave theory. ${ }^{19}$ Nevertheless, the difference is small if the values of $J_{z}$ and $\left|J_{\perp}\right|$ are close to each other, and this is favorable for ferroelectricity to take place. It is expected that the $3 \mathrm{D}$ phase diagram is quite similar to the $2 \mathrm{D}$ one. ${ }^{11}$ For the 1D case several exact solutions are known: ${ }^{18}$ the critical line between the phase of partial magnetization and saturated $d$ - or $f$-band is $\left|B_{z}\right|=J_{z}+\left|J_{\perp}\right|$, while in the Ising limit $\left(J_{z} \gg\left|J_{\perp}\right|\right)$ the critical line between the staggered orbital ordering and the phase of partial magnetization is

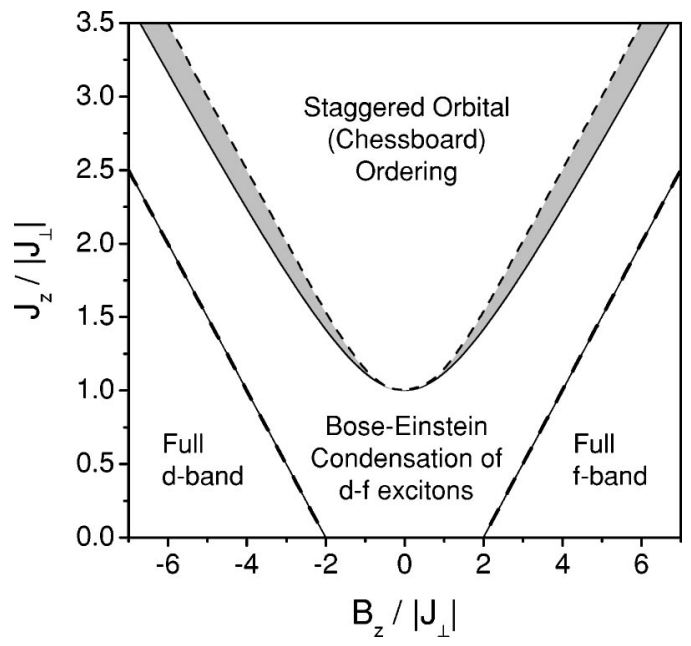

FIG. 2. Two-dimensional phase diagram of $H_{J}$ obtained from linear spin wave theory (solid lines) and from quantum Monte Carlo calculations (Refs. 11,12) (dashed lines).

$\left|B_{z}\right|=J_{z}-2\left|J_{\perp}\right|$. These relations have been reproduced in linear spin wave theory. However, since long range order does not exist in an $S=\frac{1}{2}$ Heisenberg antiferromagnetic chain where the magnetization is associated with defects in the cooperative spin-singlet ground state,${ }^{20}$ there would be a crossover region between the cooperative spin-singlet state on the small $J_{z} /\left|J_{\perp}\right|$ side and the staggered orbital ordering in the Ising limit, ${ }^{21}$ and there would be a transition from an Ising like phase to an incommensurate phase which replaces the BEC state in dimensions higher than one. ${ }^{20}$ As for the infinite dimension case, our semiclassical phase diagram should be very accurate because the spin fluctuations go to zero in that limit. Therefore, we conclude that linear spin wave theory is appropriate to the FKM with dimensions higher than one. We shall analyze the hole dynamics in each phase in the following:

Full f-band: $:^{22}$ The pseudospin configuration is a ferromagnetic state with all pseudospins down [Fig. 1(a)]. The pseudospin wave spectrum is $\omega_{\mathbf{q}}^{\text {full }}=B_{z}-z S\left(J_{z}+\left|J_{\perp}\right| \gamma_{\mathbf{q}}\right)$, where $\gamma_{\mathbf{q}}=\Sigma_{\delta} e^{i \mathbf{q} \cdot \delta} / z$ and $\delta$ is a unit vector connecting nearest neighbors. Since the hole motion will not change the pseudospin configuration, the hole can move freely with dispersion $\varepsilon_{\mathbf{k}}^{\text {full }}=-z t_{\downarrow} \gamma_{\mathbf{k}}$.

Ferroelectric phase: The pseudospin configuration is considered here as a uniform canted state [Fig. 1(c)]. The pseudospins are parallel, but they take new equilibrium direction which are tilted by a certain angle $\theta$ to the $z$ axis. This new axis of quantization is considered to lie in the $x-z$ plane as a result of spontaneous symmetry breaking. We perform a uniform rotation of the orbitals about the $y$ axis by $\theta$,

$$
\begin{gathered}
\widetilde{c}_{\mathbf{i} \uparrow}=\cos \frac{\theta}{2} e_{\mathbf{i} \uparrow}-\sin \frac{\theta}{2} e_{\mathbf{i} \downarrow}, \widetilde{c}_{\mathbf{i} \downarrow}=\sin \frac{\theta}{2} e_{\mathbf{i} \uparrow}+\cos \frac{\theta}{2} e_{\mathbf{i} \downarrow}, \\
\tau_{\mathbf{i}}^{x}=S_{\mathbf{i}}^{x} \cos \theta-S_{\mathbf{i}}^{z} \sin \theta, \quad \tau_{\mathbf{i}}^{z}=S_{\mathbf{i}}^{z} \cos \theta+S_{\mathbf{i}}^{x} \sin \theta,
\end{gathered}
$$

to obtain the ferromagnetic configuration $\left|\cdots S_{\mathbf{i}}^{z} S_{\mathbf{i}+1}^{z} \cdots\right\rangle$ $=|\cdots \downarrow \downarrow \cdots\rangle$ as the vacuum state. Then, we employ the 
slave-fermion formalism to cope with the constraint of no doubly occupancy. ${ }^{14}$ Defining holon (spinless fermion) operators $h_{\mathbf{i}}$ so that $e_{\mathbf{i} \uparrow}=h_{\mathbf{i}}^{\dagger} a_{\mathbf{i}}, e_{\mathbf{i} \downarrow}=h_{\mathbf{i}}^{\dagger}$ where $a_{\mathbf{i}}=S_{\mathbf{i}}^{-}$is the hard-core boson operator, we arrive at an effective pseudospin-polaron Hamiltonian in the momentum space,

$$
H_{t}=\sum_{\mathbf{k}} \varepsilon_{\mathbf{k}} h_{\mathbf{k}}^{\dagger} h_{\mathbf{k}}+\sum_{\mathbf{k}, \mathbf{q}} M_{\mathbf{k q} \mathbf{q}} h_{\mathbf{k}}^{\dagger} h_{\mathbf{k}-\mathbf{q}} \alpha_{\mathbf{q}}+\text { h.c. },
$$

where $\alpha_{\mathbf{q}}$ 's are pseudospin wave operators, $a_{\mathbf{q}}=u_{\mathbf{q}} \alpha_{\mathbf{q}}$ $+v_{\mathbf{q}} \alpha_{-\mathbf{q}}^{\dagger}$, with dispersion $\omega_{\mathbf{q}}=z S\left(I_{\theta, \mathbf{q}}^{2}-C_{\theta}^{2} \gamma_{\mathbf{q}}^{2}\right)^{1 / 2}$. The transformation coefficients are $u_{\mathbf{q}}=\left\{\left[I_{\theta, \mathbf{q}}\left(I_{\theta, \mathbf{q}}^{2}-C_{\theta}^{2} \gamma_{\mathbf{q}}^{2}\right)^{-1 / 2}\right.\right.$ $+1] / 2\}^{1 / 2}$ and $v_{\mathbf{q}}=-\operatorname{sgn}\left(C_{\theta} \gamma_{\mathbf{q}}\right)\left\{\left[I_{\theta, \mathbf{q}}\left(I_{\theta, \mathbf{q}}^{2}-C_{\theta}^{2} \gamma_{\mathbf{q}}^{2}\right)^{-1 / 2}\right.\right.$ $-1] / 2\}^{1 / 2}$. Here the shorthand notations are $B_{\theta}$ $=\left(-\left|J_{\perp}\right| \cos ^{2} \theta+J_{z} \sin ^{2} \theta-\left|J_{\perp}\right|\right) / 2, \quad C_{\theta}=B_{\theta}+\left|J_{\perp}\right|$, and $I_{\theta, \mathbf{q}}$ $=\left|J_{\perp}\right|+B_{\theta} \gamma_{\mathbf{q}}$. Hence $\omega_{\mathbf{q}=\mathbf{0}}=0$. The bare hole dispersion is $\varepsilon_{\mathbf{k}}=-z\left(t_{\downarrow} \cos ^{2}(\theta / 2)+t_{\uparrow} \sin ^{2}(\theta / 2)\right) \gamma_{\mathbf{k}}$, and the holepseudospin-wave coupling is

$$
M_{\mathbf{k q}}=\sin \theta \frac{t_{\uparrow}-t_{\downarrow}}{2} \frac{z}{\sqrt{N}}\left(\gamma_{\mathbf{k}-\mathbf{q}} u_{\mathbf{q}}+\gamma_{\mathbf{k}} v_{\mathbf{q}}\right) .
$$

When both pseudospin flavors have the same hopping integral, i.e., $t_{\uparrow}=t_{\downarrow}=t, H_{t}$ has the $\mathrm{SU}(2)$ symmetry, leading to a vanishing $M_{\mathbf{k q}}$ and a free hole propagation with dispersion $-z t \gamma_{\mathbf{k}}$. Hence, the fact that $t_{\uparrow} \neq t_{\downarrow}$ accounts for the formation of the pseudospin-polaron even in the presence of a ferromagnetic pseudospin background.

Using the self-consistent Born approximation (SCBA) in which the spectral functions of one hole in a $t-J$-like model can be accurately calculated, ${ }^{14-17}$ we compute the hole Green's function $G(\mathbf{k}, \omega)=\left[\omega-\varepsilon_{\mathbf{k}}-\Sigma(\mathbf{k}, \omega)+i 0^{+}\right]^{-1}$ selfconsistently with the self-energy,

$$
\Sigma(\mathbf{k}, \omega)=\sum_{\mathbf{q}} M_{\mathbf{k q}}^{2} G\left(\mathbf{k}-\mathbf{q}, \omega-\omega_{\mathbf{q}}\right) .
$$

Thus, the spectral functions are given by $A(\mathbf{k}, \omega)$ $=-\operatorname{Im} G(\mathbf{k}, \omega) / \pi$, and the quasiparticle dispersion is $E_{\mathbf{k}}$ $\equiv \varepsilon_{\mathbf{k}}+\operatorname{Re} \Sigma\left(\mathbf{k}, E_{\mathbf{k}}\right)$.

Antiferroelectric phase: This phase [Fig. 1(d)] is possible when $J_{\perp}>0$. Note that the $J_{\perp}>0$ case can be mapped onto the $J_{\perp}<0$ case by a rotation of the pseudospins in the $B$ sublattice by $180^{\circ}$ about the $z$ axis, that is, by a canonical transformation $U=\exp \left(i \Sigma_{j} \mathbf{Q} \cdot \mathbf{r}_{j} \tau_{j}^{z}\right)$, where $\mathbf{Q}=(\pi, \ldots, \pi)$. Therefore, linear spin wave theories of the antiferroelectric and ferroelectric canted states are formally connected with a displacement of momentum: $\mathbf{q} \rightarrow \mathbf{q}-\mathbf{Q}$. The effective Hamiltonian for hole hopping is

$$
H_{t}=\sum_{\mathbf{k}} \varepsilon_{\mathbf{k}} h_{\mathbf{k}}^{\dagger} h_{\mathbf{k}}+\sum_{\mathbf{k}, \mathbf{q}} M_{\mathbf{k q}} h_{\mathbf{k}}^{\dagger} h_{\mathbf{k}-\mathbf{q}} \alpha_{\mathbf{q}-\mathbf{Q}}+\text { h.c. }
$$

where $\varepsilon_{\mathbf{k}}=-z\left(t_{\downarrow} \cos ^{2}(\theta / 2)-t_{\uparrow} \sin ^{2}(\theta / 2)\right) \gamma_{\mathbf{k}}$ and

$$
M_{\mathbf{k q}}=-\sin \theta \frac{t_{\uparrow}+t_{\downarrow}}{2} \frac{z}{\sqrt{N}}\left(\gamma_{\mathbf{k}-\mathbf{q}} u_{\mathbf{q}-\mathbf{Q}}+\gamma_{\mathbf{k}} v_{\mathbf{q}-\mathbf{Q}}\right) .
$$

Likewise, we employ (7) to calculate the hole self-energy.
Staggered orbital ordering: The pseudospin configuration is a $z$-directional Néel state [Fig. 1(b)]. Considering the Néel state as the vacuum state, we define holon operators $h_{\mathbf{i}}$ and $g_{\mathbf{i}}$ so that $\widetilde{c}_{\mathbf{i} \downarrow}=h_{\mathbf{i}}^{\dagger}, \widetilde{c}_{\mathbf{i} \uparrow}=h_{\mathbf{i}}^{\dagger} a_{\mathbf{i}}$ on the $\downarrow$ sublattice and $\widetilde{c}_{\mathbf{j} \downarrow}$ $=g_{\mathbf{j}}^{\dagger} b_{\mathbf{j}}, \widetilde{c}_{\mathbf{j} \uparrow}=g_{\mathbf{j}}^{\dagger}$ on the $\uparrow$ sublattice. Here $a_{\mathbf{i}}=\tau_{\mathbf{i}}^{-}$on the $\downarrow$ sublattice and $b_{\mathbf{j}}=\tau_{\mathbf{j}}^{+}$on the $\uparrow$ sublattice are hard-core boson operators. We arrive at an effective pseudospin-polaron Hamiltonian,

$$
H_{t}=\sum_{\mathbf{k}, \mathbf{q}}^{\prime} M_{\mathbf{k q}} h_{\mathbf{k}}^{\dagger} g_{\mathbf{k}-\mathbf{q}} \alpha_{\mathbf{q}}+L_{\mathbf{k} \mathbf{q}} g_{\mathbf{k}}^{\dagger} h_{\mathbf{k}-\mathbf{q}} \beta_{\mathbf{q}}+\text { h.c., }
$$

where $\alpha_{\mathbf{q}}$ and $\beta_{\mathbf{q}}$ are pseudospin wave operators, $a_{\mathbf{q}}=u_{\mathbf{q}} \alpha_{\mathbf{q}}$ $+v_{\mathbf{q}} \beta_{-\mathbf{q}}^{\dagger}$ and $b_{-\mathbf{q}}=u_{\mathbf{q}} \beta_{-\mathbf{q}}+v_{\mathbf{q}} \alpha_{\mathbf{q}}^{\dagger}$, with dispersion $\omega_{\mathbf{q}}^{ \pm}$ $=z S\left(J_{z}^{2}-J_{\perp}^{2} \gamma_{\mathbf{q}}^{2}\right)^{1 / 2} \pm B_{z}$, respectively, and $u_{\mathbf{q}}=\left\{\left[z S J_{z} /\right.\right.$ $\left.\left.\left(\omega_{\mathbf{q}}^{+}+\omega_{\mathbf{q}}^{-}\right)+1\right] / 2\right\}^{1 / 2}, \quad v_{\mathbf{q}}=-\operatorname{sgn}\left(J_{\perp} \gamma_{\mathbf{q}}\right)\left\{\left[z S J_{z} /\left(\omega_{\mathbf{q}}^{+}\right.\right.\right.$ $\left.\left.\left.+\omega_{\mathbf{q}}^{-}\right)-1\right] / 2\right\}^{1 / 2}$. The hole-pseudospin-wave coupling is $\quad M_{\mathbf{k q}}=-z \sqrt{2 / N}\left(t_{\uparrow} \gamma_{\mathbf{k}-\mathbf{q}} u_{\mathbf{q}}+t_{\downarrow} \gamma_{\mathbf{k}} v_{\mathbf{q}}\right) \quad$ and $\quad L_{\mathbf{k q}}$ $=-z \sqrt{2 / N}\left(t_{\downarrow} \gamma_{\mathbf{k}-\mathbf{q}} u_{\mathbf{q}}+t_{\uparrow} \gamma_{\mathbf{k}} v_{\mathbf{q}}\right)$. The summations over $\mathbf{k}$ and $\mathbf{q}$ are restricted inside the reciprocal Brillouin zone of one sublattice. Within the SCBA, we obtain the following self-consistent equations for the two types of hole propagators, $G_{j}(\mathbf{k}, \omega)=\left[\omega-\Sigma_{j}(\mathbf{k}, \omega)+i 0^{+}\right]^{-1}$, where $j=g, h$ with $\Sigma_{h}(\mathbf{k}, \omega)=\Sigma_{\mathbf{q}}{ }^{\prime} M_{\mathbf{k q}}^{2} G_{g}\left(\mathbf{k}-\mathbf{q}, \omega-\omega_{\mathbf{q}}^{+}\right)$and $\Sigma_{g}(\mathbf{k}, \omega)$ $=\Sigma_{\mathbf{q}}{ }^{\prime} L_{\mathbf{k q}}^{2} G_{h}\left(\mathbf{k}-\mathbf{q}, \omega-\omega_{\mathbf{q}}^{-}\right)$.

To facilitate EFE, the system must be in a mix-valence regime and the two bands involved should have different parity, thus $t_{\uparrow}$ and $t_{\downarrow}$ have opposite signs, so do $J_{z}$ and $J_{\perp}$; furthermore, it would be more probable if both bands have similar bandwidths. ${ }^{11}$ Hence, we adopt the following parameters: ${ }^{23} t_{\uparrow}=1, t_{\downarrow}=-0.8, U^{f d}=10$, hence $J_{z}=2\left(t_{\uparrow}^{2}\right.$ $\left.+t_{\downarrow}^{2}\right) / U^{f d}=0.328$ and $J_{\perp}=4 t_{\uparrow} t_{\downarrow} / U^{f d}=-0.32$, with $B_{z}$ $=\epsilon_{d}-\epsilon_{f}$ being a free parameter-in a real material, changing $B_{z}$ could be achieved by applying pressure or alloying. Our numerical results are illustrated in the two-dimensional case. In this case, the system transits from staggered orbital ordering to the BEC of $d-f$ excitons at $\left|B_{z}\right|=0.144$ and then to a nonmixed-valence regime at $\left|B_{z}\right|=1.296$ as $B_{z}$ increases. All numerical calculations were carried out on a $16 \times 16$ square lattice.

In Figs. 3, 4, and 5, we show the spectral functions and the quasiparticle dispersions in the ferroelectric regime with $B_{z}=0.2,0.8$, and 1.2 , respectively, ${ }^{24}$ obtained from using Eq. (5). First of all, as presented in Figs. 3(a), 4(a), and 5(a), the quasiparticle bandwidth $W$ and the pseudospin wave bandwidth $W_{\text {spw }}$ have similar values. Such band narrowing can be understood in the following way: gapless pseudospin excitations are easily stimulated by incoherent hole motion, leading to the formation of the quasiparticle $(\mathrm{QP})$, pseudospin polaron, which is the propagating hole surrounded by a cloud of polarized pseudospin waves. Therefore, $W$ does not scale with hopping integrals but with $W_{\text {spw }}$.

As displayed in Figs. 3(b) $-3(d)$, for any $\mathbf{k}$, there is a well-defined quasiparticle pole (i.e., zero pseudospin wave) at the low energy side which is well separated from a broad, incoherent, multiple-pseudospin-wave background extending to the full free-electron bandwidth. Figure 3(a) shows that the bottom and the top of the QP band locate at $(\pi / 2, \pi / 2)$ 

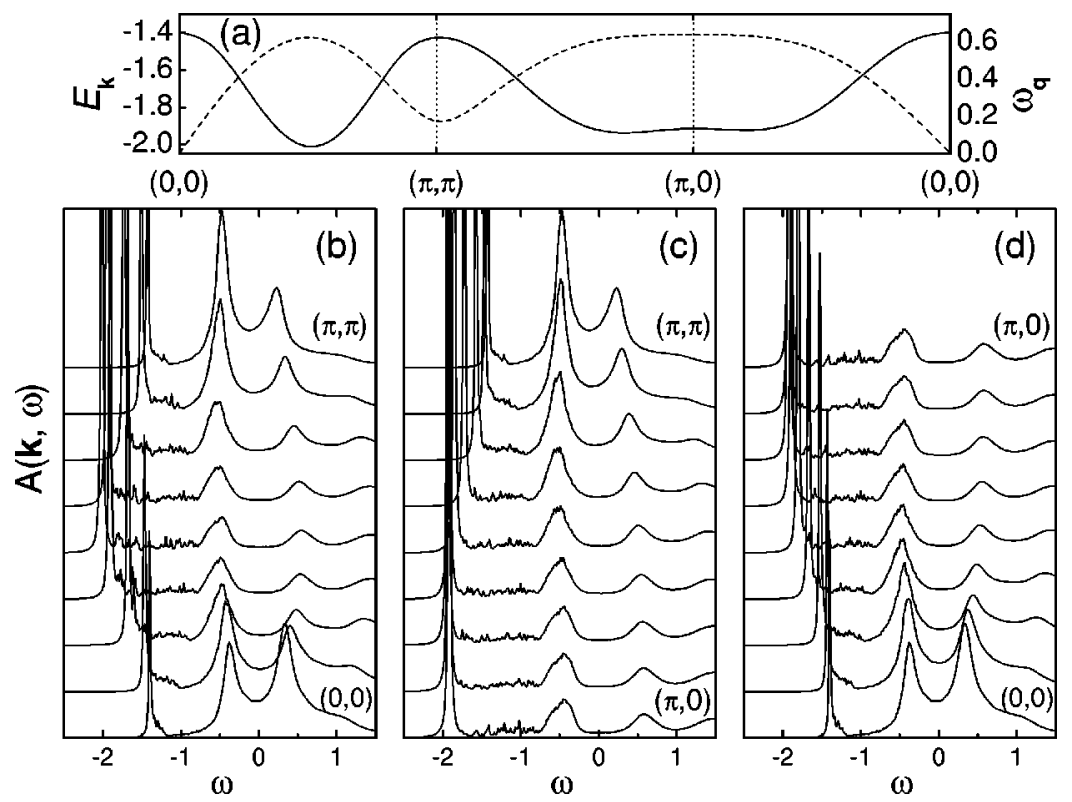

FIG. 3. (a) Presents the quasiparticle dispersion $E_{\mathbf{k}}$ (solid line, left scale), and pseudospin wave dispersion $\omega_{\mathbf{q}}$ (dashed line, right scale) in the ferroelectric phase for $B_{z}=0.2$. (b) $-(\mathrm{d})$ Shows the hole spectral functions $A(\mathbf{k}, \omega)$ along (b) $(0,0)-(\pi, \pi), \quad$ (c) $(\pi, \pi)-(\pi, 0)$, and (d) $(\pi, 0)-(0,0)$ directions.

and $(0,0)$, respectively. Furthermore, there is a flat region around $(\pi, 0)$ at which the QP energy is close to its minimum, leading to a strongly distorted density of states with a massive peak near the bottom of the QP band. These features obtained for single hole motion in the ferromagnetic pseudospin background are quite similar to those in the cuprate $t-J$ model where the hopping integrals for both spin flavors are the same and the spin background is a Heisenberg antiferromagnet. ${ }^{15}$ Note that the pseudospin wave spectrum does not vanish at $(\pi, \pi)$ while it is gapless at $(0,0)$. Consequently, the QP energy at $(\pi, \pi)$ is lower than that at $(0,0)$.

Further increasing $B_{z}$ will continue lowering the QP energy at $(\pi, \pi)$. When $B_{z}=0.8,(\pi, \pi)$ becomes the QP band bottom, as shown in Fig. 4(a). While the flat region around $(\pi, 0)$ is shrinking, a new, surprising flat region appears around $(\pi, \pi)$. Therefore, the feature of an extended van Hove singularity near the bottom of the QP band survives up to $B_{z}=0.8$. In Figs. 4(b) $-4(\mathrm{~d})$, the spectral functions are still characterized by sharp peaks at the low energy side which is well separated from a broad, incoherent background. However, apart from the QP band bottom, considerable spectral weights move to the incoherent part, indicating that the hole motion is more severely damped by incoherent processes.

When $B_{z}$ is increased to 1.2 [Figs. 5(b) $-5(\mathrm{~d})$ ], almost all spectral weights locate in the incoherent part except near the QP band bottom, $(\pi, \pi)$. This means that the hole spectral weights are strongly reduced by a cloud of polarized pseudospin waves. The shape of the QP dispersion [Fig. 5(a)] becomes similar to that of free hole dispersion $\propto \gamma_{\mathbf{k}}$, yet with bandwidth $W \simeq W_{\text {spw }}$. As $B_{z}$ further increases, the spectral weights at wave vectors not very close to $(\pi, \pi)$ goes to zero quickly until the pseudospin polaron picture becomes invalid at $B_{z}=1.296$. Then, the system evolves into full

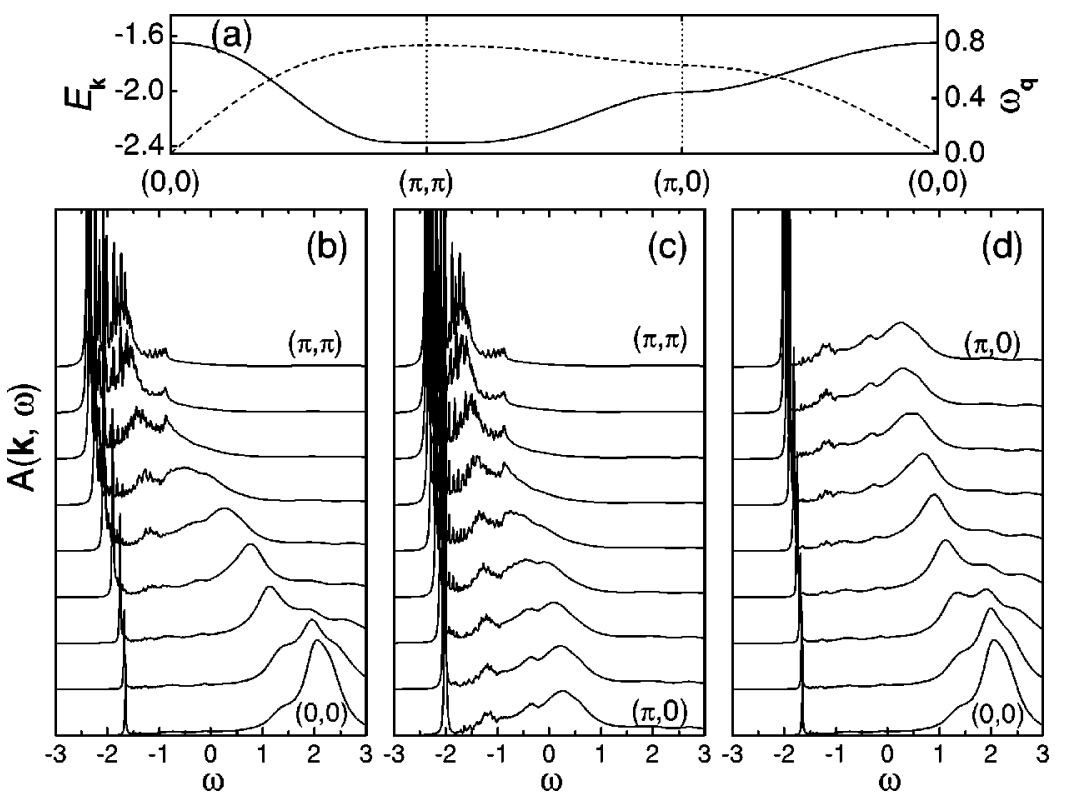

FIG. 4. The same quantities as in Fig. 3 but for $B_{z}=0.8$. 


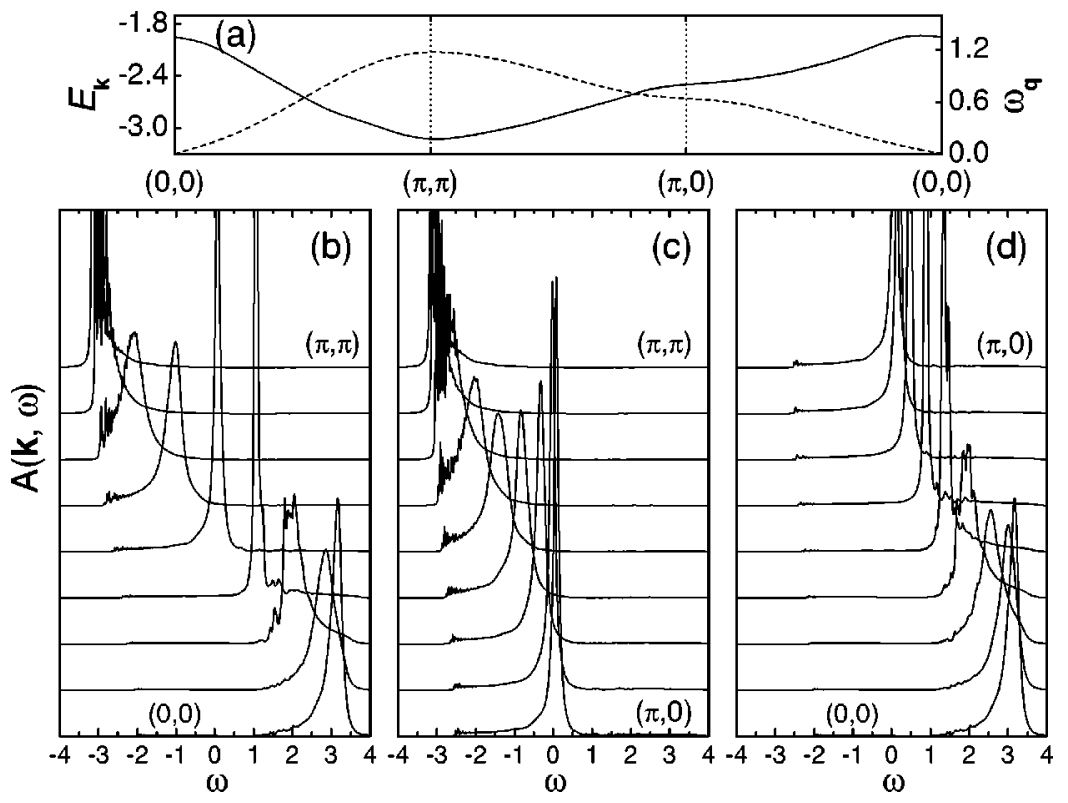

FIG. 5. The same quantities as in Fig. 3 but for $B_{z}=1.2$. $f$-band with free hole motion, implying a unit spectral weight everywhere.

To summarize, we present a systematic study on the evolution of the spectral functions of a single hole in the FKM as a function of $B_{z}$, the $d$ - and $f$-level energy difference. We find that strong scattering processes between the hole and $d-f$ excitons exist in the mix-valence regimes, thus lead to the formation of pseudospin polarons and interesting electronic structures with bandwidth scaling with that of the pseudospin excitation, even for single hole propagation in a ferromagnetic pseudospin background (i.e., the ferroelectric phase) due to the different hopping integrals of the $d$ - and $f$-electrons. Furthermore, flat regions near the bottom of the quasiparticle band are found in a wide range of $B_{z}$ in the two-dimensional case. As we know, similar anomalous, yet crucial hole spectral features were found in high temperature superconductors, ${ }^{13,15}$ thus we speculate that besides EFE, the extended Falicov-Kimball model could have many novel properties, especially upon hole doping.

We are grateful to C. D. Batista for stimulating discussions. This work is supported by the Nebraska Research Initiative, the Nebraska EPSCOR-NSF Grant EPS-9720643, and Department of the Army Grants DAAG 55-98-1-0273 and DAAG 55-99-1-0106. W.N.M. is grateful for the support from the Office of Naval Research.
*Electronic mail address: wgyin@yahoo.com

${ }^{1}$ C. Kittel, Introduction to Solid State Physics (Wiley, New York, 1996).

${ }^{2}$ T. Portengen, T. Östreich, and L.J. Sham, Phys. Rev. Lett. 76, 3384 (1996).

${ }^{3}$ T. Portengen, T. Östreich, and L.J. Sham, Phys. Rev. B 54, 17452 (1996).

${ }^{4}$ L.M. Falicov and J.C. Kimball, Phys. Rev. Lett. 22, 997 (1969).

${ }^{5}$ J.K. Freericks and V. Zlatić, cond-mat/0301188.

${ }^{6}$ G. Czycholl, Phys. Rev. B 59, 2642 (1999).

${ }^{7}$ P. Farkašovský, Phys. Rev. B 59, 9707 (1999).

${ }^{8}$ P. Farkašovský, Phys. Rev. B 65, 081102(R) (2002).

${ }^{9}$ V. Zlatić, Philos. Mag. B 81, 1443 (2001).

${ }^{10}$ L.G. Sarasua and M.A. Continentino, Phys. Rev. B 65, 233107 (2002).

${ }^{11}$ C.D. Batista, Phys. Rev. Lett. 89, 166403 (2002).

${ }^{12}$ G. Schmid, S. Todo, M. Troyer, and A. Dorneich, Phys. Rev. Lett. 88, 167208 (2002).

${ }^{13}$ E. Dagotto, A. Nazarenko, and A. Moreo, Phys. Rev. Lett. 74, 310 (1995)
${ }^{14}$ S. Schmitt-Rink, C.M. Varma, and A.E. Ruckenstein, Phys. Rev. Lett. 60, 2793 (1988).

${ }^{15}$ W.-G. Yin, C.D. Gong, and P.W. Leung, Phys. Rev. Lett. 81, 2534 (1998).

${ }^{16}$ W.-G. Yin, H.Q. Lin, and C.D. Gong, Phys. Rev. Lett. 87, 047204 (2001).

${ }^{17}$ J. Bala, G.A. Sawatzky, A.M. Oleś, and A. Macridin, Phys. Rev. Lett. 87, 067204 (2001).

${ }^{18}$ C.N. Yang and C.P. Yang, Phys. Rev. 151, 258 (1966).

${ }^{19}$ We are grateful to C.D. Batista for correcting an incorrect statement on this point in an earlier version of this paper.

${ }^{20}$ D.C. Dender, P.R. Hammar, D.H. Reich, C. Broholm, and G. Aeppli, Phys. Rev. Lett. 79, 1750 (1997).

${ }^{21}$ A. Langari, Phys. Rev. B 58, 14467 (1998).

${ }^{22}$ The phase diagram of $H_{J}$ is symmetric under a change of sign of $B_{z}$ because $H_{J}$ is symmetric under a reflection in the $x-y$ plane.

${ }^{23}$ These parameters are close to the realistic ones for cuprates and manganites, see Refs. 15,16.

${ }^{24}$ The results in staggered orbital ordering are similar to those in the ferroelectric phase with $B_{z}=0.2$. 